\title{
Mathematical modeling, numerical simulation and experimental comparison of the desorption process in a metal hydride hydrogen storage system
}

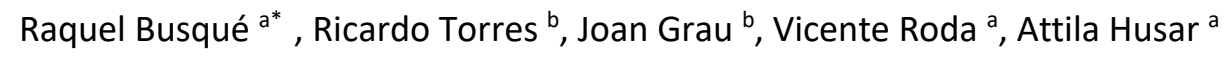 \\ a Institut de Robòtica i Informàtica Industrial, CSIC-UPC Llorens i Artigas 4-6, 08028 Barcelona, Spain \\ ${ }^{b}$ Fluid Mechanics Department. EEBE UPC - Barcelona Tech. C/ Eduard Maristany, 10-14, 08019 Barcelona, Spain
}

\begin{abstract}
A two-dimensional axisymmetric model is developed to study the hydrogen desorption reaction and its subsequent discharge in a metal hydride canister. Experimental tests are performed on an in-house fabricated setup. An extensive study on the effects of the metal properties and boundary conditions on discharging performance is carried out through nondestructive testing (NDT). Results show that the desorption process is more effective if the activation energy for desorption $\left(E_{d}\right)$ and the reaction enthalpy $(\Delta H)$ decrease, and when the desorption rate coefficient $\left(C_{d}\right)$ and the external convection heat transfer coefficient when the bottle is being heated $(h)$ increase. Furthermore, porosity $(\varepsilon)$ can be useful for the design of hydrogen storage systems, with a trade-off between charge/discharge time and storage capacity. Numerical and experimental results are compared achieving a good agreement. These results can be used to select metal hydride materials and also for the future evaluation of metal hydride degradation.
\end{abstract}

Keywords: Hydrogen storage; Metal hydride; Hydrogen desorption; Two-dimensional axisymmetric simulation; Experimental testing

\section{Nomenclature}

$\begin{array}{llll}C_{a} & \text { Absorption rate constant }\left[\mathrm{s}^{-1}\right] & \text { Subscripts } & \\ C_{d} & \text { Desorption rate constant }\left[\mathrm{s}^{-1}\right] & a & \text { Absorption } \\ c_{p} & \text { Heat capacity }\left[\mathrm{Kg}^{-1} \mathrm{~K}^{-1}\right] & \mathrm{amb} & \text { Ambient } \\ E_{a} & \text { Activation energy for absorption }\left[\mathrm{kJ} \mathrm{mol}^{-1}\right] & \mathrm{atm} & \text { Atmospheric } \\ E_{d} & \text { Activation energy for desorption }\left[\mathrm{kJ} \mathrm{mol}^{-1}\right] & \mathrm{B} & \text { Bottom } \\ f_{L} & \text { Pressure loss coefficient }\left[\mathrm{kg} \mathrm{m}^{-4} \mathrm{~S}^{-1}\right] & \mathrm{d} & \text { Desorption } \\ \vec{g} & \text { Gravity }\left[\mathrm{m} \mathrm{s}^{-2}\right] & \mathrm{emp} & \text { Empty } \\ h & \text { Convection coefficient }\left[\mathrm{W} \mathrm{m}^{-2} \mathrm{~K}^{-1}\right] & \mathrm{h} & \text { Heater } \\ H / M & \text { Hydrogen to metal ratio } & \text { ini } & \text { Initial } \\ k & \text { Thermal conductivity }\left[\mathrm{W} \mathrm{m} \mathrm{m}^{-1} \mathrm{~K}^{-1}\right] & L & \text { Lateral } \\ K & \text { Permeability }\left[\mathrm{m}^{2}\right] & \text { ref } & \text { Reference }\end{array}$

\footnotetext{
*Corresponding autor: Raquel Busqué (rbusque@iri.upc.edu)

Tel: +34934015751

Fax: +34934015750

Co-authors: Ricardo Torres (ricardo.torres@upc.edu)

Joan Grau (joan.grau@upc.edu)

Vicente Roda (vroda@iri.upc.edu)

Attila Husar (ahusar@iri.upc.edu)
} 


$\begin{array}{llll}M & \text { Molecular weight }\left[\mathrm{kg} \mathrm{mol}^{-1}\right] & \text { sat } & \text { Saturation } \\ \vec{n} & \text { Normal vector } & & \\ p & \text { Pressure }[\mathrm{Pa}] & \text { Superscripts } & \\ p_{e q} & \text { Equilibrium pressure }[\mathrm{Pa}] & \text { eff } & \text { Effective } \\ R & \text { Universal gas constant }\left[\mathrm{J} \mathrm{mol}^{-1} \mathrm{~K}^{-1}\right] & g & \text { Gas } \\ S_{m} & \text { Mass source/sink term }\left[\mathrm{kg} \mathrm{m}^{-3} \mathrm{~s}^{-1}\right] & \mathrm{m} & \text { Metal } \\ S_{T} & \text { Energy source } / \text { sink term }\left[\mathrm{W} \mathrm{m}^{-3}\right] & & \\ t & \text { Time }[\mathrm{s}] & & \\ T & \text { Temperature }[\mathrm{K}] & \\ \vec{u} & \text { Velocity field }\left[\mathrm{m} \mathrm{s}^{-1}\right] & \\ & & \\ G r e e k s & & \\ \Delta H & \text { Reaction enthalpy }\left[\mathrm{kJ} \mathrm{mol}^{-1}\right] & \\ \Delta S & \text { Entropy }\left[\mathrm{J} \mathrm{mol}^{-1} \mathrm{~K}^{-1}\right] & \\ \varepsilon & \text { Porosity } & \\ \theta & \text { Absorbed hydrogen fraction } & \\ \mu & \text { Dynamic viscosity }\left[\mathrm{kg} \mathrm{m}^{-1} \mathrm{~s}^{-1}\right] & \\ \rho & \text { Density }\left[\mathrm{kg} \mathrm{m}^{-3}\right] & \end{array}$

\section{Introduction}

In the last decades, the fossil fuel consumption has been steadily increasing and so, society, industry and governments have become aware of the necessities to invest in sustainable energies to reduce the carbon emissions. Recent studies [1] show that the use of hydrogen as an energy vector can aid to satisfy the present and future energy demands without additional carbon emissions.

Hydrogen has been considered a good, carbon-free alternative to hydrocarbon fuels, as it is abundant in water and could be easily produced using renewable energy sources. Hence, it can facilitate the transition from the present fossil fuel energy economy to a future hydrogen based economy $[2,3]$.

A major concern that needs to be addressed to make hydrogen technology economically feasible is the safe storage of hydrogen in vessels with the desirable weight, volume and cost. The future of hydrogen as an energy vector will strongly depend on these technologies [4].

Hydrogen storage techniques have been widely evaluated in recent years, and have been categorized in three main areas [5]:

1. High pressure gaseous hydrogen storage

2. Liquefaction of hydrogen gas and liquid-phase hydrogen storage

3. Material-based hydrogen storage, including: sorbents, chemical hydrogen storage materials and metal hydrides

Hydrogen stored as a absorbed element inside metal hydride materials offers certain advantages compared to high-pressure gaseous or cryogenic liquid storage systems in terms of compactness, storage at conditions close to ambient, energy required to store the hydrogen, possibility of tailoring metal hydrides to suit different temperature-pressure requirements, as 
well as being inherently safe because hydrogen is stored at low pressure [6-8]. The disadvantage is that it offers low energetic density per unit of mass due to the weight of the metal itself. But on the contrary, it offers high energy density per unit of volume.

A lot of studies have been made regarding the modeling of hydrogen storage in metal hydride systems, but most of these studies are focused on hydrogen absorption reactions [2,6,8-20], and numerical studies of the hydrogen desorption process are relatively rare $[3,14,19-27]$.

Prior studies show that reactions in metal beds can be effectively modeled as a twodimensional problem. Jemni and Ben Nasrallah [12,22] developed two-dimensional heat and mass transfer models for hydrogen absorption and desorption respectively. Their results show that the difference between the solid and hydrogen temperatures is negligible, except for some limited areas close to the gas outlet and tank wall, so the local thermal equilibrium hypothesis can be used. In a subsequent study, Jemni et al. [28] experimentally determined the effective thermal conductivity, the equilibrium pressure and the reaction kinetics, as well as the temperature and the pressure over time. Then, they tested the validity of the theoretical model comparing it to the experimental results achieving a good agreement between all the data.

Regarding desorption, the main interest has been the enhancement of the reaction focusing on its thermal behavior. For this purpose, Chung et al. [14] developed a two-dimensional model and investigated the effects of heat convection on the charging/discharging performance. Both Darzi et al. [19] and Mellouli et al. [21], analyzed the cooling and heating of a cylindrical LaNi $\mathrm{Li}_{5}$ metal hydride tank together with some kind of heat exchanger. The firsts ones, used an annular jacket filled with a phase change material (PCM), and the latter ones compared three different heat exchanger designs. They concluded that a properly designed heat exchanger can reduce the hydrogen charging/discharging times considerably. Moreover, Delavar et al. [24], numerically studied effects of parameters such as: heating fluid temperature around the tank, hydrogen outlet pressure, and heat transfer coefficient between heating fluid and the metal hydride storage tank during the hydrogen release. On the other hand, Wang et al. [29] studied two different scenarios during absorption, positioning the metal hydride bottle vertically or horizontally on the model. Unfortunately, neither Chung et al. [14], Darzi et al. [19], Mellouli et al. [21], Delavar et al. [24] nor Wang et al. [29] provided a comparison of the hydrogen desorption simulations with experiments.

Additionally, Kyoung at al. [26] developed a three-dimensional metal hydride model to be used for the design of industrial metal hydride storage vessels. The model was validated against the experimental data reported by Jemni et al. [28] achieving a good agreement. Mohammadshahi et al. [30] also focused on a practical application. They improved the metal hydride model to incorporate several features, such as: temperature variation of the cooling fluid or introduction of the non-ideal gas equation.

Regarding experimental results, Dhaou et al. [15] empirically studied hydriding/dehydriding rates and the pressure-composition isotherms for different metal hydrides under quasiisothermal and variable pressure conditions, and finally they derived empirical rate equations to describe the sorption reaction kinetics. However, they did not obtain experimental data at system level. 
In a previous work, Busqué et al. [8], the effects of different metal properties and other parameters were tested and analyzed during hydrogen absorption in a metal hydride storage system both numerically and experimentally. Numerical and experimental data achieved a good agreement, and therefore, the results can be used to identify different behaviors of the metal hydride material or evaluate its current status.

However, although considerable efforts have been made to model the hydrogen desorption process in metal hydride storage vessels, most of these models have not been validated against experimental data and they do not consider the whole application both numerically and experimentally. Furthermore, a detailed analysis of the desorption and discharge processes and their complex transport mechanisms, and mass and heat transfer is in great demand for an efficient design, and selection of materials for any industrial application involving metal hydride based hydrogen storage.

This work provides an extensive study on the hydrogen desorption process and its subsequent discharge in a metal hydride storage system. The effects of each individual metal property through non-destructive testing (NDT) during desorption have been studied as well. It is also important to mention that a detailed experimental setup has been designed and assembled emulating a real application using commercially available metal hydride canisters to obtain real data at system level, which has not been widely investigated in prior studies.

To that end, a 2D axisymmetric numerical model has been developed and some parametric studies have been carried out to obtain the effect of the abovementioned properties on the desorption and discharging performance at system level. In other words, it gives an idea of how the hydrogen desorption and discharge can be improved depending on the properties of the selected metal hydride material. Then, these results have been used to calibrate the simulation to the real practical application. Next, the outcomes have been compared with experimental data obtained in an in-house fabricated setup. The model and the experimental protocols can be used to estimate the metal hydride internal state as well as evaluating aspects such as, the reduction of the storage capacity due to contamination. This study is meaningful as a guide towards the engineering practice of the desorption process of the metal hydride hydrogen storage technology.

\section{Mathematical and numerical model}

A two-dimensional axisymmetric metal hydride hydrogen storage container is modeled to analyze the key physical phenomena occurring during the hydrogen desorption process. A 2D axisymmetric model is used, which intends not only to achieve the geometrical symmetry of the bottle but also the symmetry of the metal hydride inside, as well as all its physical and morphological properties.

The hydrogen-metal reaction is the following:

$$
M+\frac{x}{2} H_{2} \leftrightarrow M H_{x}+\Delta H
$$


Where $M$ represents the metal, $M H_{x}$ is the respective hydride and $x$ the ratio of hydrogen to metal. Finally, $\Delta H$ is the heat of reaction. The hydride formation is exothermic and consequently the reverse reaction is endothermic.

The modeled geometry has a bottle-like shape (Fig. 1) that matches the real metal hydride vessels available in the lab. The geometry is implemented as a two-dimensional axisymmetric domain, which has two differentiated sections: the top section is a void region acting as a buffer for the hydrogen molecules. This section is drawn as a quarter of a circle of radius 23 $\mathrm{mm}$ plus a small tube in the center which is the outlet area, being $16.10 \mathrm{~mm}$ long and 3.18 $\mathrm{mm}$ wide. The larger bottom section is a porous region which contains the metal hydride and where the absorption/desorption reactions take place. This domain is basically a rectangle of $273.25 \mathrm{~mm}$ long and $23 \mathrm{~mm}$ wide.

To deal with the lack of information regarding the metal hydride inside the canisters, a detailed and systematic numerical analysis of various metal properties is performed.

This model is implemented using the commercially available software COMSOL Multiphysics ${ }^{\circledR}$, by means of the appropriate fluid dynamics and heat transfer modules.

\subsection{Model assumptions}

The assumptions made in the model are the following:

1. Hydrogen is in a gaseous state and is considered to be an ideal gas.

2. Powdery metal hydride is treated as an isotropic and homogeneous porous medium.

3. Local thermal equilibrium is assumed between the solid metal and the hydrogen gas. This means that the gas and metal local temperatures inside the vessel will be the same.

4. Volumetric compression of the metal hydride during desorption is neglected.

5. Metal hydride properties such as porosity, permeability and thermal conductivity remain constant during the desorption process.

\subsection{Conservation equations and source terms}

Under the assumptions above, the metal hydride container is governed by the conservation of mass, momentum and thermal energy $[14,17,18,26]$.

\subsubsection{Mass conservation}

For hydrogen:

$$
\varepsilon \frac{\partial \rho^{g}}{\partial t}+\nabla\left(\rho^{g} \vec{u}\right)=-S_{m}
$$

Where the gas density can be described by the ideal gas law.

For the metal hydride:

$$
(1-\varepsilon) \frac{\partial \rho^{m}}{\partial t}=S_{m}
$$




\subsubsection{Momentum conservation}

In the porous region, the momentum conservation is modeled using a continuum approach through the Brinkman equations.

$$
\frac{\rho^{g}}{\varepsilon} \frac{\partial \vec{u}}{\partial t}=-\nabla p+\nabla \tau-\frac{\mu}{K} \vec{u}-\frac{S_{m}}{\varepsilon^{2}} \vec{u}+\rho^{g} \vec{g}
$$

In the hydrogen buffer area Navier-Stokes equation considering laminar flow (5) is used instead.

$$
\rho \frac{\partial u}{\partial t}=\nabla\left[-p I+\mu\left(\nabla u+(\nabla u)^{T}\right)-\frac{2}{3} \mu(\nabla u) I\right]+F
$$

\subsubsection{Energy conservation}

Regarding the temperature field, as local thermal equilibrium is assumed between the gas phase and the solid metal hydride, the energy equation can be expressed with a single temperature variable as follows [31]:

$$
\overline{\rho c_{p}} \frac{\partial T}{\partial t}+\nabla\left(\rho^{g} c_{p}^{g} \vec{u} T\right)=\nabla\left(k^{e f f} \nabla T\right)+S_{T}
$$

In Eq. (6), the effective heat capacity $\left(\overline{\rho c_{p}}\right)$, and the effective thermal conductivity $\left(k^{e f f}\right)$ can be expressed as porosity-weighted functions of the hydrogen and metal phases.

$$
\begin{gathered}
\overline{\rho c_{p}}=(1-\varepsilon) \rho^{m} c_{p}^{m}+\varepsilon \rho^{g} c_{p}^{g} \\
k^{e f f}=(1-\varepsilon) k^{m}+\varepsilon k^{g}
\end{gathered}
$$

\subsubsection{Source terms and equilibrium pressure}

The energy source term (9) represents the heat loss during the endothermic hydrogen desorption reaction.

$$
S_{T}=S_{m}\left[\Delta H-T\left(c_{p}^{g}-c_{p}^{m}\right)\right]
$$

The mass source/sink term is defined by equations (10), (11) and (12). The reaction proceeds to the right to form the metal hydride or to the left to release hydrogen depending on the equilibrium pressure. To ensure that the numerical model fulfills the equilibrium conditions and decides accordingly to absorb or desorb, some constrains are added:

- If $p^{g}>p_{e q_{a}}:$ Absorption

$$
S_{m}=C_{a} \exp \left(-\frac{E_{a}}{R T}\right) \ln \left(\frac{p^{g}}{p_{e q_{a}}}\right)\left(\rho_{s a t}^{m}-\rho^{m}\right)
$$


- If $p_{e q_{a}}>p^{g}>p_{e q_{d}}$

$$
S_{m}=0
$$

- If $p^{g}<p_{e q_{d}}$ : Desorption

$$
S_{m}=C_{d} \exp \left(-\frac{E_{d}}{R T}\right) \ln \left(\frac{p^{g}-p_{e q_{d}}}{p_{e q_{d}}}\right)\left(\rho^{m}-\rho_{e m p}^{m}\right)
$$

The equilibrium of the reaction can also change depending on the temperature. If additional heat is supplied, the system wants to achieve equilibrium temperature and shifts to the left, desorbing and being endothermic to recover equilibrium conditions. On the contrary, if heat is removed from the system (for example a fan is connected), the reaction compensates this change in temperature proceeding to the right, enhancing the exothermic absorption process.

In Fig. 2, the slope of the isotherm plateau and the hysteresis between absorption and desorption isotherms are inherent characteristics and vary among different metal hydrides. These also depend on experimental variables such as pressure, temperature and mates. florthe $L_{a N i_{5}}-H_{2}$ system, the hysteresis is $\ln \left(\frac{p_{e q a}}{p_{e q_{d}}}\right)=0.13$ [14].

The equilibrium pressure for hydrogen desorption on $\mathrm{LaNi}_{5}$ has been measured experimentally and it approximates $f(H / M)$ as a ninth-order polynomial function as shown in equation (13)

[15].

$$
\begin{aligned}
p_{e q_{d}} & =p_{\text {ref }} \exp \left(\frac{\Delta H}{R T_{\text {ref }}}-\frac{\Delta S}{R}\right)=\left(a_{0}+\sum_{n=1}^{9} a_{n}\left(\frac{H}{M}\right)^{n}\right) \exp \left(\frac{\Delta H}{R}\left(\frac{1}{T}-\frac{1}{T_{\text {ref }}}\right)\right) \\
a_{0} & =0.421 \quad a_{1}=-4.114 \quad a_{2}=14.180 \quad a_{3}=-13.109 \quad a_{4}=4.557 \\
a_{5} & =0.166 \quad a_{6}=-0.592 \quad a_{7}=0.179 \quad a_{8}=-0.023 \quad a_{9}=1.126 E-3
\end{aligned}
$$

On the other hand, several other researchers $[14,19,24,32]$ have used a simplified version of the equilibrium pressure, called Van't Hoff equation (left hand side of equation (13)). The Van't Hoff equation in this form is only valid for a limited temperature range in which $\Delta H$ does not change much with temperature.

Finally, hydrogen to metal ratio is defined by equation (14), and the desorbed hydrogen fraction $(\theta)$ can be defined according to equation (15), where $(H / M)_{\text {sat }}$ is the saturated hydrogen to metal atomic ratio. Therefore $\theta$ ranges from 0 to 1 .

$$
(H / M)=\frac{2\left(\rho^{m}-\rho_{e m p}^{m}\right) / M_{H 2}}{\rho_{e m p}^{m} / M_{m}}
$$




$$
\theta=\frac{(H / M)}{(H / M)_{s a t}}
$$

\subsection{Initial/boundary conditions and model implementation}

At first, the metal hydride container is assumed to be in thermodynamic equilibrium, so the initial conditions are the following:

$$
T=T_{\text {ini }} \quad p=p_{\text {ini }} \quad \rho^{m}=\rho_{\text {ini }}^{m} \quad \vec{u}=0
$$

Where $\rho_{i n i}^{m}$ represents the initial metal hydride density, which may be equal to the hydrogen saturated metal hydride density or lower if the metal is only partially filled.

As for boundary conditions (Fig. 1), boundary walls are assumed to be impermeable so, no-slip velocity and no-flux conditions are valid and a convection boundary condition is applied between the bottle walls and exterior air.

$$
k^{e f f} \frac{\partial T}{\partial \vec{n}}=h\left(T_{h}-T\right)
$$

Besides the convection with surrounding air, a thin stainless-steel layer has been added to the exterior boundaries, emulating the metallic container. The thickness is $2.4 \mathrm{~mm}$ and its thermal conductivity is specified considering stainless steel 304L (UNSS30403) as a material.

Hydrogen is discharged to the atmosphere, so pressure at the outlet has been set to atmospheric. However, it is important to note that the whole experimental system is being considered, so the exiting hydrogen mass flow circulates through a circuit that generates pressure losses and then reaches the exterior (atmospheric pressure). To emulate that, a pressure loss coefficient is set at the outlet $\left(f_{L}\right)$. This pressure gradient between the interior of the bottle and the exterior drives the evacuation of hydrogen from the bottle.

\section{Experimental characterization}

In order to compare the simulations with the experimental results and also to observe the evolution of the parameters in a practical application, the following experimental setup is implemented $[8,13,23]$.

As seen in Fig. 3, during discharge (red circuit), hydrogen leaves the selected bottle, which are Swagelok 304L-HDF4-500-PD stainless steel containers filled with an unknown metal hydride. Then, the discharge pressure is measured using the pressure sensor $\mathrm{P} 1$. This sensor is an industrial pressure transmitter from Impress Sensors and Systems (IMP-G4002-7A4-BCV-00000) working with pressures up to $4 \mathrm{MPa}$. Then, hydrogen circulates through an alternative circuit and goes through a 7-micron particulate filter, to ensure that no particles enter the MFC. And then it goes into an EL-FLOW base Mass Flow Controller F-201C-AAA-22V (MFC) from Bronkhorst High Tech which has a range from from 0 to $1.5 \mathrm{nlpm}$. Finally, hydrogen is released to the atmosphere. At the outer surface of the bottles there are also some welded tip PFA thermocouples from tc direct (T1 to T8) attached in order to acquire temperature data. The control and data acquisition system is programmed in LabVIEW, and the communication 
between the analogical and digital signals is achieved using the National Instruments (NI) myRIO-1900 device.

Additionally, a heater is connected to heat up the bottles and enhance the desorption reaction. The heater is a hot air blower HOTWIND SYSTEM from Leister Technologies AG. At the outlet of this air blower a flexible tube and a PVC pipe are connected. The metal hydride bottle perfectly fits inside the PVC pipe, and so the canister walls are heated (Fig. 4).

Through this experimental setup, temperatures at the surface of the bottles, pressure at the outlet and exiting mass flow can be measured and then used to calibrate the parameters of the computational simulation.

In order to perform the experiments, the following protocols have been executed. First, it is necessary that the bottle that is going to be studied in each of the experiments is charged or partially charged with hydrogen. Also, it is important that all valves are closed before starting the process.

Once the setup protocol is completed, the discharging process can start. First, the valve corresponding to the selected bottle is opened, being it V1 or V2. Then, V4 is opened in the direction that allows hydrogen flow towards V6. Then, $\mathrm{V} 6$ is opened and hydrogen goes towards the MFC. Finally, the outlet of the system is opened using V5.

At this instant the bottle is in discharging mode, and the system will continue working until the hydrogen discharge curve is saturated, which means that it has reached a steady asymptotic situation in which a relatively small amount of hydrogen is released (lower than $0.01 \mathrm{nlpm}$ ). At this point, the bottle is considered empty.

When the bottle is empty, discharge process is completed and so valve V5, followed by V 6 and V4 are closed. Finally, the bottle valve (V1 or V2) is closed.

\section{Results and discussion}

\subsection{Closed bottle equilibrium results}

To verify that the numerical model is able to reproduce equilibrium conditions some simulations considering that the bottle is closed and the metal is partially filled with hydrogen are performed.

As the metal inside the bottle is unknown, it is decided to use literature data, corresponding to $\mathrm{LaNi}_{5}$, shown in Table 1 to perform the first simulations. Equilibrium pressure is taken from the polynomial fitting of equation (13).

Fig. 5 represents absolute pressure and temperature evolutions considering that the outlet is assumed to be closed. In Fig. 5 a) and b), a situation in which the initial gas pressure inside the container is higher than the equilibrium pressure at ambient temperature. As the system wants to reach equilibrium conditions, it absorbs some free hydrogen molecules, increasing its temperature as the reaction is exothermic, and reaching equilibrium. 
Fig. 5 c) and d), represent the opposite scenario, in which the gas pressure is lower than the equilibrium pressure at ambient temperature. In this case the system desorbs some of the hydrogen to increase the pressure inside the bottle and reach equilibrium. Temperature decreases as the desorption reaction is endothermic.

\subsection{Limitations and unknowns}

That being said, there have been some limitations when trying to represent the numerical model to match experimental conditions that make it difficult to compare experimental and simulation data.

It is important to mention that the metal hydride bottles used in this study are commercially available, and the provider is not willing to disclose information regarding the type of metal hydride. As the container is sealed, it is not possible to determine the material properties needed to run the simulations without having to open up the canister directly. This would be a destructive test, which is not an option.

Besides not knowing the metal hydride, its current state, quality or degree of degradation are unknown as well.

As the bottles are closed and so it is impossible to see inside, the morphology and internal distribution, porosity, permeability or available storage space are also unknown. It is also unknown if there is a hydrogen buffer region, the size of it, and also if there is some kind of manifold or other device that distributes the hydrogen within the bottle during charge or discharge.

There are some parameters that can be obtained by theoretical calculations or from the experimental tests and measurements. These parameters are: heating temperature $\left(T_{h}\right)$, initial pressure $\left(p_{\text {ini }}\right)$, initial and saturated metal hydride density $\left(\rho_{\text {ini }}^{m}, \rho_{\text {sat }}^{m}\right)$, lateral and bottom convection heat transfer coefficient $\left(h_{L}, h_{B}\right)$ and pressure loss coefficient $\left(f_{L}\right)$, and are specified in Table 2 [23].

Regarding equilibrium pressure, it has been decided to use the Van't Hoff equation to study the hydrogen desorption and compare it to experimental values, as the metal is unknown, and therefore a semi-empirical adjustment for it is not available.

\subsection{Sensitivity of the model to metal properties, structural parameters and working conditions}

One objective of this work is to determine the material properties without performing ex situ material testing, so an in-situ strategy is presented.

It is important to mention that computational models allow studying scenarios that may be difficult or even impossible to obtain experimentally. For example, the variation of $C_{d}$ and $E_{d}$ independently from one another might not have a physical meaning, as they are both dependent on the metal hydride. However, it is very interesting to visualize any specific behavior when varying input variables as an identification mechanism, as well as its effect on discharging performance. 
So, a parametric study is performed to identify the sensitivity of the model to the parameters that influence the metal hydride. Using this data, the model is calibrated by isolating the specific material properties that are more sensitive to the model.

Fig. 6 a) and b) show that the desorption process becomes more effective if $E_{d}$ decreases, which makes sense, as it is easier for the system to overcome that activation energy for the desorption process to start if its value is lower. This can be contrasted with the values of pressure (Fig. 6 a)), which are higher as $E_{d}$ decreases. This means that the pressure gradient is larger and hydrogen will tend to desorb, and so the system's temperature decreases (Fig. 6 b)) as the endothermic reaction is more effective.

In Fig. 6 c) and d) the desorption reaction is more effective when $C_{d}$ increases, as it generates a higher desorption rate, according to equation (12).

In Fig. 6 e) and f), pressure increases as $\Delta H$ decreases, and consequently the mass flow increases as well. Regarding temperature, and according to equation (9), as $\Delta H$ increases, $S_{T}$ increases as well and so the temperature is lower.

It is important to mention that in the published literature, the value of $E_{d}$ usually varies between 13000 and $59371.6 \mathrm{~J} \mathrm{~mol}^{-1}$, the value of $C_{d}$ usually varies between 0.043 and $2127.3 \mathrm{~s}$ ${ }^{1}$ and the value of $\Delta H$ usually varies between 17600 and $64230.4 \mathrm{~J} \mathrm{~mol}^{-1}$, for different metal hydride materials $[2,9,14,19,20,24-27,33]$.

Focusing on porosity (Fig. $6 \mathrm{~g}$ ) and $\mathrm{h}$ )), it is important to bear in mind that the volume of the bottle does not change, and so, when porosity is high $(\varepsilon=0.7)$ there is less metal inside the bottle, and consequently less absorbed hydrogen stored. As the amount of hydrogen stored is lower, the desorption process finishes sooner and the temperature increases until it reaches ambient temperature. On the other hand, when the metal is not very porous $(\varepsilon=$ 0.4 ), the desorption process is slower; as there is a lot more metal inside the bottle and so more hydrogen is stored. The effect of the porosity on the metal hydride behavior can be useful for the design of hydrogen storage systems, with a trade-off between charge/ discharge time and storage capacity.

In Fig. 6 i) and j), it can be seen that externally heating the system has a huge impact on desorption performance, although in this case $T_{h}$ is $297 \mathrm{~K}$. At higher convection heat transfer coefficients $(h)$, the process becomes more effective. Thus, pressure and temperature inside the bottle increase and desorbed hydrogen fraction decreases.

The values of porosity usually vary between 0.4 and 0.63 in the current literature, and regarding convection a local heat transfer coefficient of up to $10 \mathrm{~W} \mathrm{~m}^{-2} \mathrm{~K}^{-1}$ represents the scenario in which the tank is heated by natural convection, whilst $10<h<200 \mathrm{~W} \mathrm{~m}^{-2} \mathrm{~K}^{-1}$ represent forced air heating scenarios and finally $h>200 \mathrm{~W} \mathrm{~m}^{-2} \mathrm{~K}^{-1}$ represents liquid heating scenarios.

We are aware that the overall results of this study are not independent on the selected initial metal. However, these parametric studies can be used, from an engineering point of view, to determine how the desorption and discharge performance can be improved depending on the 
metal hydride properties, or in more general terms, depending on the selected metal hydride material for a given application.

\subsection{Simulation results and experimental data comparison}

In Fig. 7, shows the results both experimentally and numerically. The model has been calibrated using the parameters in Table 2, A for Bottle 1 and Table 2, B for

Bottle 2.

Fig. 7 a), show the comparison between experimental pressure measured outside the bottle and simulation results modeled inside the bottle. A sharp decrease on the pressure is observed at the beginning, as the hydrogen that was not absorbed and is stored in gas form comes out quickly, producing a huge drop in pressure. Then, the absorbed hydrogen inside the metal starts to desorb, increasing the internal pressure. Finally, pressure progressively decreases as there is no more hydrogen inside the bottle until it reaches atmospheric conditions. As can be seen, both curves match, and the small deviations are a consequence of the pressure difference by pressure losses, as the simulated pressure is calculated inside the bottle and the measurements are taken outside of the bottle, plus the many uncertainties explained throughout the paper.

On Fig. $7 \mathrm{~b}$ ), the temperature evolution at the surface of the bottle is depicted. As a heater is being used, temperature on the surface of the bottles increases. Again, it reaches a kind of plateau or small slope curve region, as there is a balance between the endothermic reaction and the heat supplied, and finally, when the reaction has finished, the temperature increases according to the set point temperature of the heater. In this case, temperature is not a good indicator of the desorption reaction, because a heater is being used and so the temperature measurement and the simulation results at the surface of the bottle basically give information on the heater temperature. That being said, both curves match well and so the validity of the model is checked. It also has to be taken into account that Bottle 1 had a larger amount of hydrogen stored, so the discharge process takes longer.

\section{Conclusions}

A mathematical model, which rigorously accounts for the principles of mass, momentum and energy conservation, and absorption/desorption kinetics, is developed to simulate the hydrogen desorption process in a metal hydride tank, and its subsequent discharge at system level.

The results of these parametric studies have allowed the identification of the parameters that most influence the system's behavior, thus achieving an adequate calibration of the model. It has been concluded that the desorption process is more effective if the activation energy for desorption $E_{d}$ and reaction enthalpy $\Delta H$ decrease, and if desorption rate coefficient $C_{d}$ increases. As expected, the process is also enhanced if the convection heat transfer coefficient $h$ is increased when the bottle is being heated. Regarding porosity, the value of $\varepsilon$ can be useful for the design of hydrogen storage systems, with a trade-off between charge/ discharge time and storage capacity.

It is important to highlight the experimental contribution of this paper using commercially available metal hydride bottles. The whole system is designed, assembled and a data 
acquisition system using a NI myRIO device is programmed on LabVIEW, to obtain the required experimental data to be compared with the numerical model.

During discharge, the pressure curve follows an exponential relaxation at the beginning until it reaches a plateau, as the reaction is enhanced and the released hydrogen molecules inside the bottle increase the internal pressure. Then, pressure progressively decreases as there is less hydrogen inside the bottle, until it reaches atmospheric conditions. Regarding temperature, it is difficult to compare simulation and experimental results, as the thermocouples are placed at the outer surface of the bottle, being very sensitive to the heater temperature.

All in all, the simulation results show the same tendencies in comparison with experimental data, and so it is demonstrated that the model successfully captures the essential trends that occur in the system.

The results and observations of this paper can be used for the characterization of a metal hydride storage system. Therefore, an effective design of the storage system can be implemented. The analysis of the metal properties can be used as a tool for selecting metal hydride materials, or in other words, it can give an idea of how the hydrogen desorption and discharge can be improved depending on the properties of the selected metal hydride material.

Besides that, the results can also be used for monitoring the current status of the bottle and the supervision of its performance, evaluating aspects such as degradation of the metal hydride material due to fatigue or wear, or reduction of the hydrogen absorption capacity of the metal due to contamination (for example presence of air and water vapor). Additionally, as the model is able to reproduce absorption and desorption phenomena, it can be used to model cyclic charge and discharge processes, as it is likely that a real application would use a hydrogen storage system cyclically.

In conclusion, this study is meaningful as a guide towards the engineering practice of the desorption process of the metal hydride hydrogen storage technology.

\section{Acknowledgements}

All the experimental tests were performed at the PEM Fuel Cells Laboratory of the Institut de Robòtica i Informàtica Industrial (CSIC-UPC, Barcelona, Spain) and was only possible due to its advanced equipment and proficient technical staff. This work has been partially funded by the Spanish national project MICAPEM (ref. DPI2015-69286-C3-2-R, MINECO/FEDER) and the Spanish State Research Agency through the María de Maeztu Seal of Excellence to IRI MDM2016-0656.

\section{References}

[1] Sharaf OZ, Orhan MF. An overview of fuel cell technology : Fundamentals and applications. Renew Sustain Energy Rev 2014;32:810-53. doi:10.1016/j.rser.2014.01.012.

[2] Muthukumar P, Singhal A, Bansal GK. Thermal modeling and performance analysis of industrial-scale metal hydride based hydrogen storage container. Int J Hydrogen Energy 2012;37:14351-64. doi:10.1016/j.ijhydene.2012.07.010. 
[3] Dhaou H, Mellouli S, Askri F, Jemni A, Ben Nasrallah S. Experimental and numerical study of discharge process of metal-hydrogen tank. Int J Hydrogen Energy 2007;32:1922-7. doi:10.1016/j.ijhydene.2006.08.045.

[4] Rusman NAA, Dahari M. A review on the current progress of metal hydrides material for solid-state hydrogen storage applications. Int J Hydrogen Energy 2016;41:12108-26. doi:10.1016/j.ijhydene.2016.05.244.

[5] Fuel Cells Technologies Office. Hydrogen Storage. US Dep Energy 2017. https://energy.gov/eere/fuelcells/hydrogen-storage (accessed September 5, 2017).

[6] Patil SD, Ram Gopal M. Analysis of a metal hydride reactor for hydrogen storage. Int J Hydrogen Energy 2013;38:942-51. doi:10.1016/j.ijhydene.2012.10.031.

[7] Sakintuna B, Lamari-Darkrim F, Hirscher M. Metal hydride materials for solid hydrogen storage: A review. Int J Hydrogen Energy 2007;32:1121-40. doi:10.1016/j.ijhydene.2006.11.022.

[8] Busqué R, Torres R, Grau J, Roda V, Husar AP. Effect of metal hydride properties in hydrogen absorption through 2D-axisymmetric modeling and experimental testing in storage canisters. Int J Hydrogen Energy 2017;42:19114-25. doi:10.1016/j.ijhydene.2017.06.125.

[9] Akanji O, Kolesnikov A. Modeling and Simulation of Hydrogen Storage Device for Fuel Cell Using COMSOL Multiphysics. COMSOL Conf. 2011 Stuttgart, 2011, p. 1-26.

[10] Nam J, Ko J, Ju H. Three-dimensional modeling and simulation of hydrogen absorption in metal hydride hydrogen storage vessels. Appl Energy 2012;89:164-75. doi:10.1016/j.apenergy.2011.06.015.

[11] Jiao K, Li X, Yin Y, Zhou Y, Yu S, Du Q. Effects of various operating conditions on the hydrogen absorption processes in a metal hydride tank. Appl Energy 2012;94:257-69. doi:10.1016/j.apenergy.2012.01.033.

[12] Jemni A, Ben Nasrallah S. Study of two-dimensional heat and mass transfer during absorption in a metal-hydrogen reactor. Int J Hydrogen Energy 1995;20:43-52. doi:10.1016/0360-3199(93)E0007-8.

[13] Busqué R. Metal Hydride State Of Charge Estimator. Development and experimental validation of the hydrogen storage system model. Bachelor's Thesis. UPC-CSIC, 2015.

[14] Chung CA, Ho CJ. Thermal-fluid behavior of the hydriding and dehydriding processes in a metal hydride hydrogen storage canister. Int J Hydrogen Energy 2009;34:4351-64. doi:10.1016/j.ijhydene.2009.03.028.

[15] Dhaou H, Askri F, Ben Salah M, Jemni A, Ben Nasrallah S, Lamloumi J. Measurement and modelling of kinetics of hydrogen sorption by LaNi5 and two related pseudobinary compounds. Int J Hydrogen Energy 2007;32:576-87. doi:10.1016/j.ijhydene.2006.07.001.

[16] Baldissin D, Lombardo D. Thermofluidynamic Modelling of Hydrogen Absorption and Desorption in a LaNi4.8Al0.2 Hydride Bed. COMSOL Conf. 2009 Milan, 2009.

[17] Bao Z, Wu Z, Nyamsi SN, Yang F, Zhang Z. Three-dimensional modeling and sensitivity analysis of multi-tubular metal hydride reactors. Appl Therm Eng 2013;52:97-108. doi:10.1016/j.applthermaleng.2012.11.023.

[18] Talagañis BA, Meyer GO, Aguirre PA. Modeling and simulation of absorption-desorption cyclic processes for hydrogen storage-compression using metal hydrides. Int J Hydrogen Energy 2011;36:13621-31. doi:10.1016/j.ijhydene.2011.07.139.

[19] Darzi AAR, Afrouzi HH, Moshfegh A, Farhadi M. Absorption and desorption of hydrogen 
in long metal hydride tank equipped with phase change material jacket. Int J Hydrogen Energy 2016;41:9595-610. doi:10.1016/j.ijhydene.2016.04.051.

[20] Ben Mâad H, Miled A, Askri F, Ben Nasrallah S. Numerical simulation of absorptiondesorption cyclic processes for metal-hydrogen reactor with heat recovery using phasechange material. Appl Therm Eng 2016;96:267-76. doi:10.1016/j.applthermaleng.2015.11.093.

[21] Mellouli S, Askri F, Dhaou H, Jemni A, Ben Nasrallah S. Numerical study of heat exchanger effects on charge / discharge times of metal - hydrogen storage vessel. Int J Hydrogen Energy 2009;34:3005-17. doi:10.1016/j.ijhydene.2008.12.099.

[22] Jemni A, Nasrallah S Ben. Study of two-dimensional heat and mass transfer during desorption in a metal-hydrogen reactor. Int J Hydrog Energy 1995;20:881-91.

[23] Busqué R. Numerical modeling and experimental analysis of the desorption process in a metal hydride hydrogen storage system. Master's Thesis. UPC-CSIC, 2017.

[24] Valizadeh M, Delavar MA, Farhadi M. Numerical simulation of heat and mass transfer during hydrogen desorption in metal hydride storage tank by Lattice Boltzmann method. Int J Hydrogen Energy 2015;41:413-24. doi:10.1016/j.ijhydene.2015.11.075.

[25] Ben Mâad H, Askri F, Ben Nasrallah S. Numerical investigation of heat and mass transfer during the desorption process of an $\mathrm{Mg} 2 \mathrm{Ni}-\mathrm{H} 2$ reactor. Int J Hydrogen Energy 2013;38:4597-610. doi:10.1016/j.ijhydene.2013.01.144.

[26] Kyoung S, Ferekh S, Gwak G, Jo A, Ju H. Three-dimensional modeling and simulation of hydrogen desorption in metal hydride hydrogen storage vessels. Int J Hydrogen Energy 2015;40:14322-30. doi:10.1016/j.ijhydene.2015.03.114.

[27] Yoo H, Ko J, Yun S, Chang M, Kang H, Kim W, et al. A numerical investigation of hydrogen desorption phenomena in $\mathrm{ZrCo}$ based hydrogen storage beds. Int J Hydrogen Energy 2013;38:6226-33. doi:10.1016/j.ijhydene.2012.12.098.

[28] Jemni A, Ben Nasrallah S, Lamloumi J. Experimental and theoretical study of a metalhydrogen reactor. Int J Hydrog Energy1 1999;24:631-44. doi:10.1016/S03603199(98)00117-7.

[29] Wang Y, Cordobes X, Chen J, Guang X, Miller T. Three-dimensional modeling of hydrogen sorption in metal hydride hydrogen storage beds 2009;194:9971006. doi:10.1016/j.jpowsour.2009.06.060.

[30] Mohammadshahi SS, Gould T, Gray EM, Webb CJ. An improved model for metalhydrogen storage tanks e Part 1 : Model development. Int J Hydrogen Energy 2016;41:3537-50. doi:10.1016/j.jijhydene.2015.12.050.

[31] Nield D, Bejan A. Convection in Porous Media. Springer-Verlag New York; 2006. doi:10.1007/0-387-33431-9.

[32] Askri F, Jemni A, Ben Nasrallah S. Study of two-dimensional and dynamic heat and mass transfer in a metal-hydrogen reactor. Int J Hydrogen Energy 2002;28:537-57. doi:10.1016/S0360-3199(02)00141-6.

[33] Odysseos M, Rango P De, Christodoulou CN, Hlil EK, Steriotis T, Karagiorgis G, et al. The effect of compositional changes on the structural and hydrogen storage properties of (La-Ce)Ni5 type intermetallics towards compounds suitable for metal hydride hydrogen compression. J Alloys Compd 2013;580:S268-70. doi:10.1016/j.jallcom.2013.01.057. 


\begin{tabular}{|c|c|c|}
\hline Parameter & Designation & Value \\
\hline Absorption rate constant & $C$ & $59.178\left[s^{-1}\right]$ \\
\hline Desorption rate constant & $C_{d}$ & $9.57\left[s^{-1}\right]$ \\
\hline Hydrogen heat capacity & $c_{p}^{g}$ & $14890\left[\mathrm{Jg}^{-1} \mathrm{~K}^{-1}\right]$ \\
\hline Metal heat capacity & $c_{p}^{m}$ & $419\left[\mathrm{Jg}^{-1} \mathrm{~K}^{-1}\right]$ \\
\hline Activation energy for absorption & $E$ & $21179.6\left[\mathrm{~J} \mathrm{~mol}^{-1}\right]$ \\
\hline Activation energy for desorption & $E_{d}$ & $23879.6\left[\mathrm{~J} \mathrm{~mol}^{-1}\right]$ \\
\hline Pressure loss coefficient & $f_{L}$ & $2 E 10\left[\mathrm{~kg} \mathrm{~m}^{-4} \mathrm{~s}^{-1}\right]$ \\
\hline Bottom surface convection heat transfer coefficient & $h_{B}$ & $4.3\left[W m^{-2} K^{-1}\right]$ \\
\hline Lateral surface convection heat transfer coefficient & $h_{L}$ & $4.3\left[W m^{-2} K^{-1}\right]$ \\
\hline Permeability & $K$ & $1 E-8\left[m^{2}\right]$ \\
\hline Hydrogen thermal conductivity & $k^{g}$ & $0.1672\left[\mathrm{~W} \mathrm{~m}^{-1} \mathrm{~K}^{-1}\right]$ \\
\hline Metal thermal conductivity & $k^{m}$ & $1.04\left[W m^{-1} K^{-1}\right]$ \\
\hline $\mathrm{H}_{2}$ molecular weight & $M_{H 2}$ & $0.0020159\left[\mathrm{~kg} \mathrm{~mol}^{-1}\right]$ \\
\hline Metal molecular weight & $M_{m}$ & $0.432\left[\mathrm{~kg} \mathrm{~mol}^{-1}\right]$ \\
\hline Atmospheric pressure & $p_{\text {atm }}$ & $1 E 5[\mathrm{~Pa}]$ \\
\hline Initial bottle pressure & $p_{\text {ini }}$ & $3 E 5[P a]$ \\
\hline Reference pressure & $p_{\text {ref }}$ & $1 E 6[\mathrm{~Pa}]$ \\
\hline Universal gas constant & $R$ & $8.314\left[\mathrm{~J} \mathrm{~mol}^{-1} \mathrm{~K}^{-1}\right]$ \\
\hline Ambient temperature & $T_{a m b}$ & $297[K]$ \\
\hline Heating temperature & $T_{h}$ & $297[K]$ \\
\hline Initial bottle temperature & $T_{i n i}$ & $298[K]$ \\
\hline Reference temperature & $T_{\text {ref }}$ & $298[K]$ \\
\hline Reaction enthalpy & $\Delta H$ & $35620\left[\mathrm{~J} \mathrm{~mol}^{-1}\right]$ \\
\hline Reaction entropy & $\Delta S$ & $108\left[\mathrm{~J} \mathrm{~mol}^{-1} \mathrm{~K}^{-1}\right]$ \\
\hline Porosity & $\varepsilon$ & 0.5 \\
\hline Metal volume fraction & $\theta_{m}$ & 0.95 \\
\hline Hydrogen dynamic viscosity & $\mu$ & $8.411 E-6[\mathrm{~Pa} \cdot \mathrm{s}]$ \\
\hline Hydrogen-free metal density & $\rho_{e m p}^{m}$ & $5300\left[\mathrm{~kg} \mathrm{~m}^{-3}\right]$ \\
\hline Initial metal hydride density & $\rho_{i n i}^{m}$ & $5307.3\left[\mathrm{~kg} \mathrm{~m}^{-3}\right]$ \\
\hline Saturated metal hydride density & $\rho_{\text {sat }}^{m}$ & $5369\left[\mathrm{~kg} \mathrm{~m}^{-3}\right]$ \\
\hline
\end{tabular}




\begin{tabular}{|c|c|c|c|}
\hline Parameter & Designation & A. Bottle 1 & B. Bottle 2 \\
\hline Desorption rate constant & $C$ & $9.57\left[s^{-1}\right]$ & $5\left[s^{-1}\right]$ \\
\hline $\begin{array}{l}\text { Activation energy for } \\
\text { desorption }\end{array}$ & $E$ & $23880\left[\mathrm{~J} \mathrm{~mol}^{-1}\right]$ & $20000\left[\mathrm{~J} \mathrm{~mol}^{-1}\right]$ \\
\hline Pressure loss coefficient & $f_{L}$ & $2 E 10\left[\mathrm{~kg} \mathrm{~m}^{-4} \mathrm{~s}^{-1}\right]$ & $2.5 E 10\left[\mathrm{~kg} \mathrm{~m}^{-4} \mathrm{~s}^{-1}\right]$ \\
\hline $\begin{array}{l}\text { Bottom surface convection heat } \\
\text { transfer coefficient }\end{array}$ & $h_{B}$ & $70\left[W m^{-2} K^{-1}\right]$ & $70\left[W m^{-2} K^{-1}\right]$ \\
\hline $\begin{array}{l}\text { Lateral surface convection heat } \\
\text { transfer coefficient }\end{array}$ & $h_{L}$ & $20\left[W m^{-2} K^{-1}\right]$ & $20\left[W m^{-2} K^{-1}\right]$ \\
\hline Initial bottle pressure & $p_{\text {ini }}$ & $2.6 E 5[P a]$ & $2.7 E 5[P a]$ \\
\hline Ambient temperature & $T_{a m b}$ & $296.39[K]$ & $296.27[K]$ \\
\hline Heating temperature & $T_{h}$ & $324.15[K]$ & $328.15[K]$ \\
\hline Initial bottle temperature & $T_{\text {ini }}$ & $296.39[K]$ & $296.27[K]$ \\
\hline Reference temperature & $T_{\text {ref }}$ & $296.39[k]$ & $296.27[K]$ \\
\hline Porosity & $\varepsilon$ & 0.5 & 0.55 \\
\hline Initial metal hydride density & $\rho_{i n i}^{m}$ & $5320.4\left[\mathrm{~kg} \mathrm{~m}^{-3}\right]$ & $5313.5\left[\mathrm{~kg} \mathrm{~m}^{-3}\right]$ \\
\hline Saturated metal hydride density & $\rho_{\text {sat }}^{m}$ & $5334.1\left[\mathrm{~kg} \mathrm{~m}^{-3}\right]$ & $5328.7\left[\mathrm{~kg} \mathrm{~m}^{-3}\right]$ \\
\hline
\end{tabular}


Click here to download high resolution image
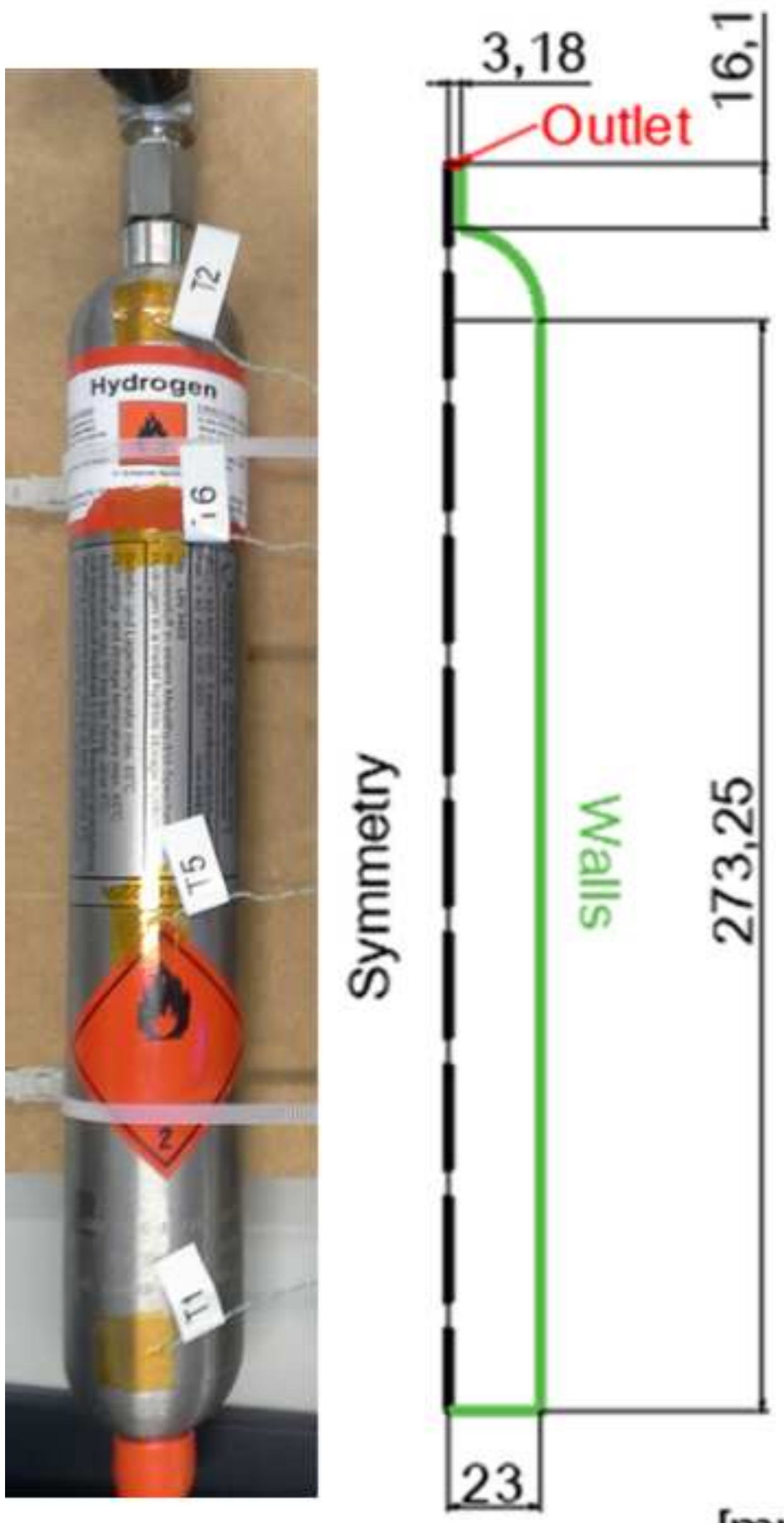

$[\mathrm{mm}]$ 
Click here to download high resolution image

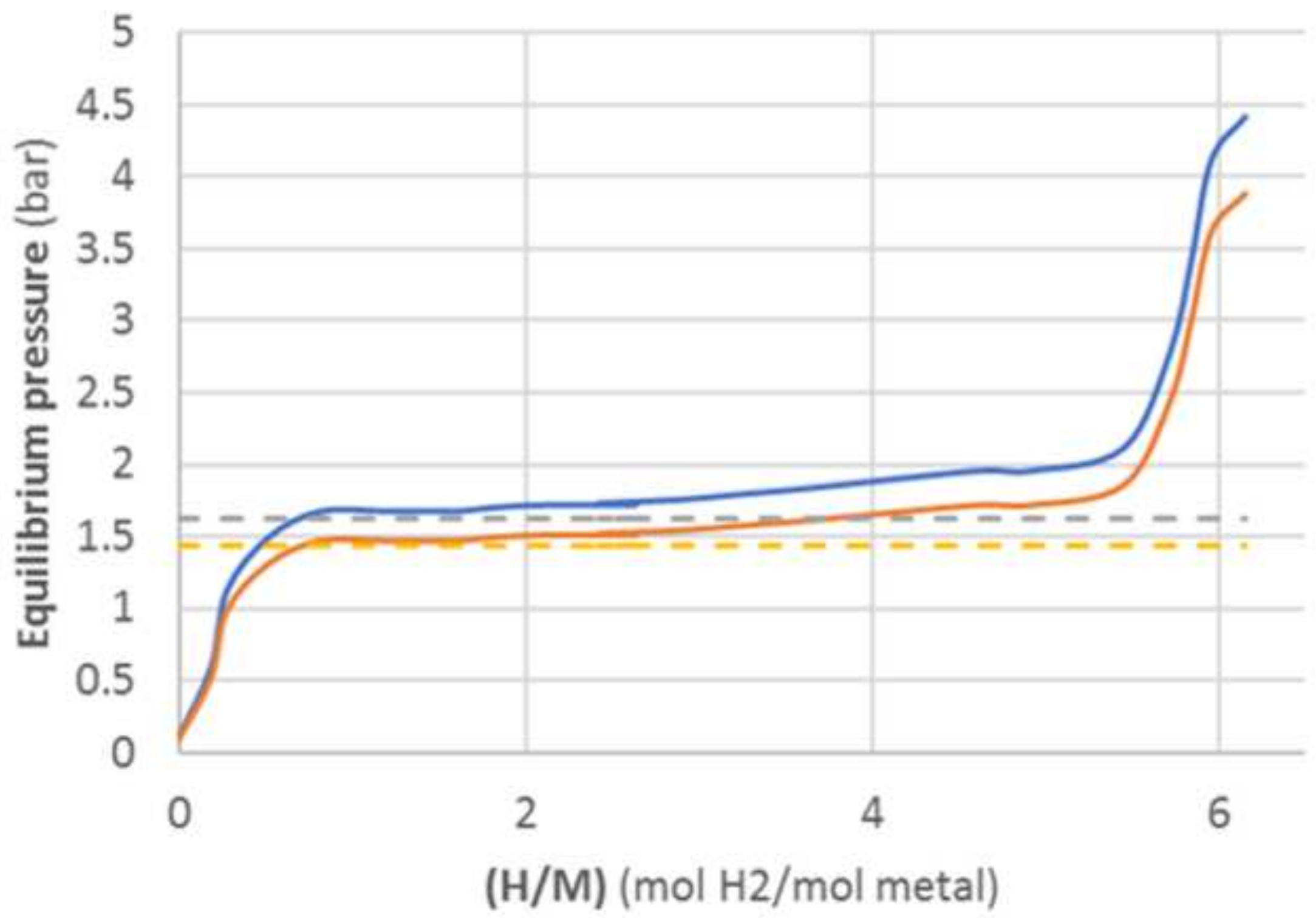

- Ads - Empirical isotherm —Des - Empirical isotherm - - - Ads - Van't Hoff equation - - - Des - Van't Hoff equation 
Click here to download high resolution image

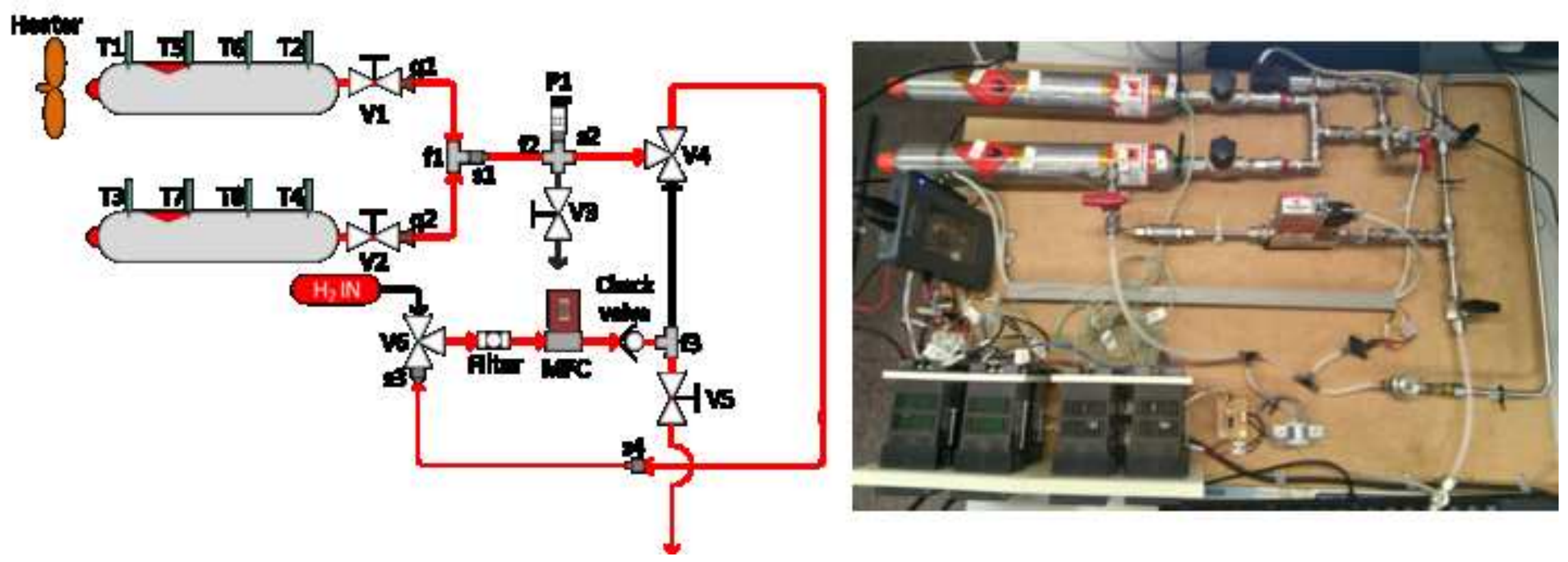


Click here to download high resolution image

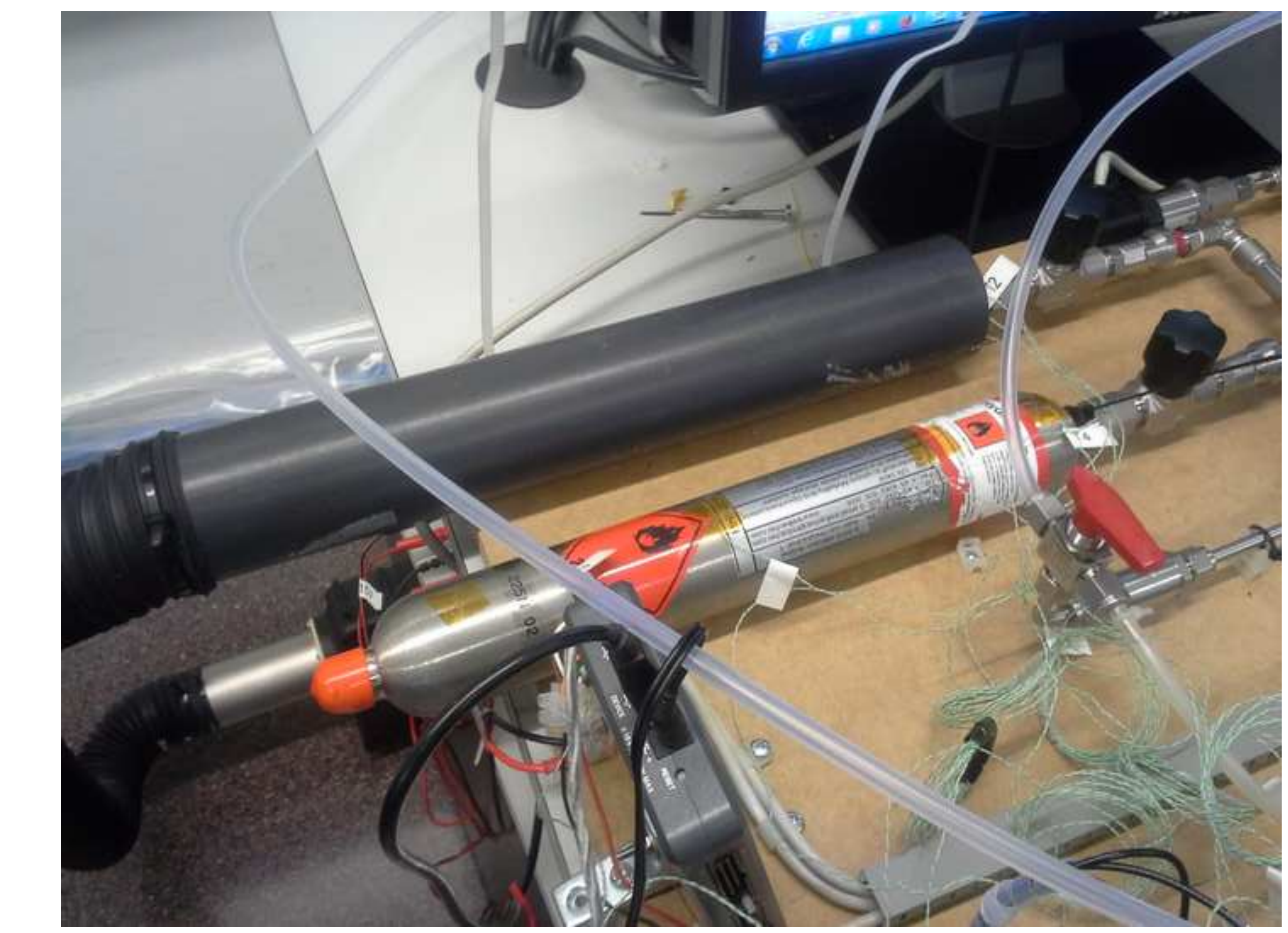


Click here to download high resolution image
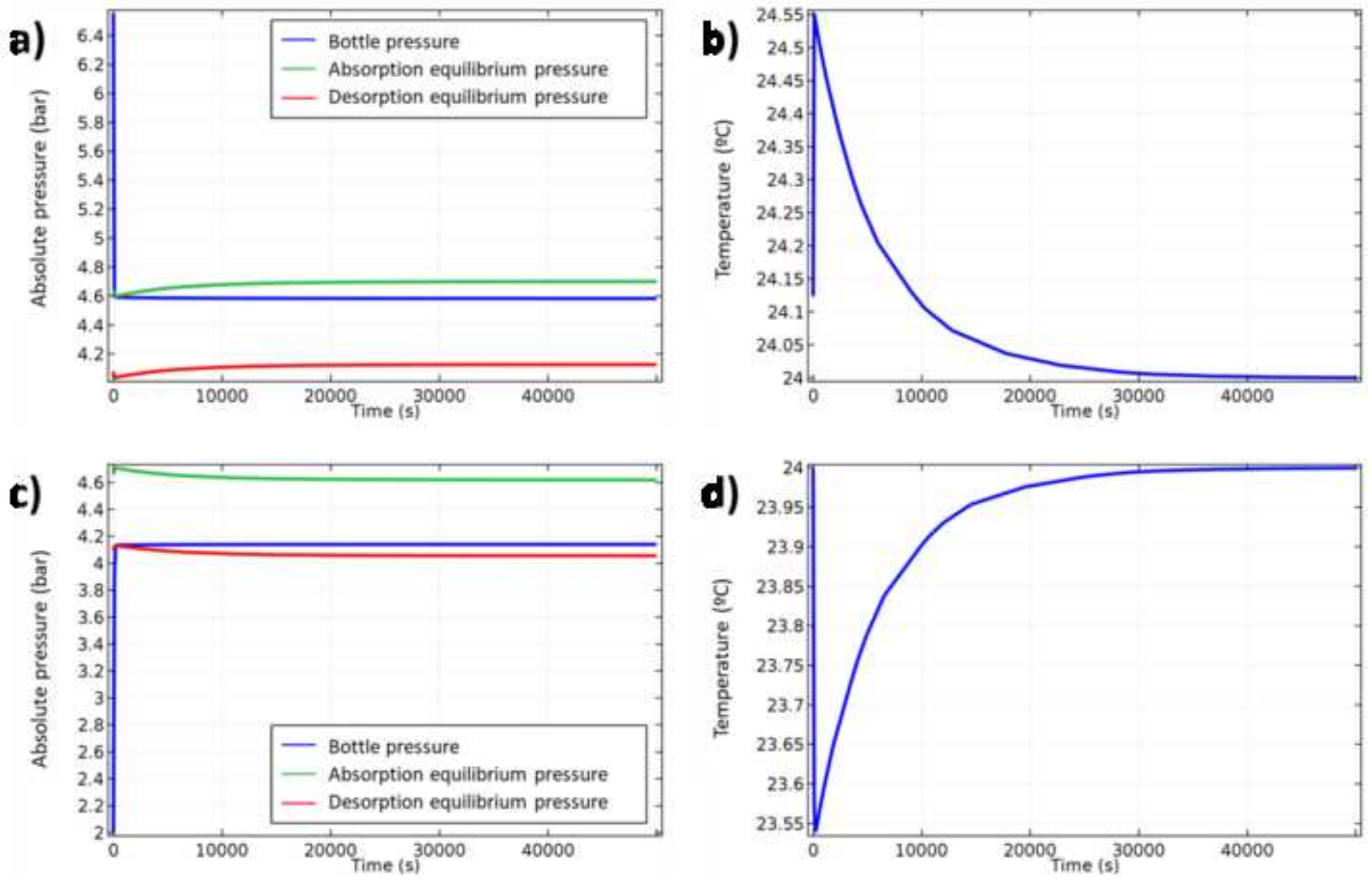


\section{Figure 6}

Click here to download high resolution image
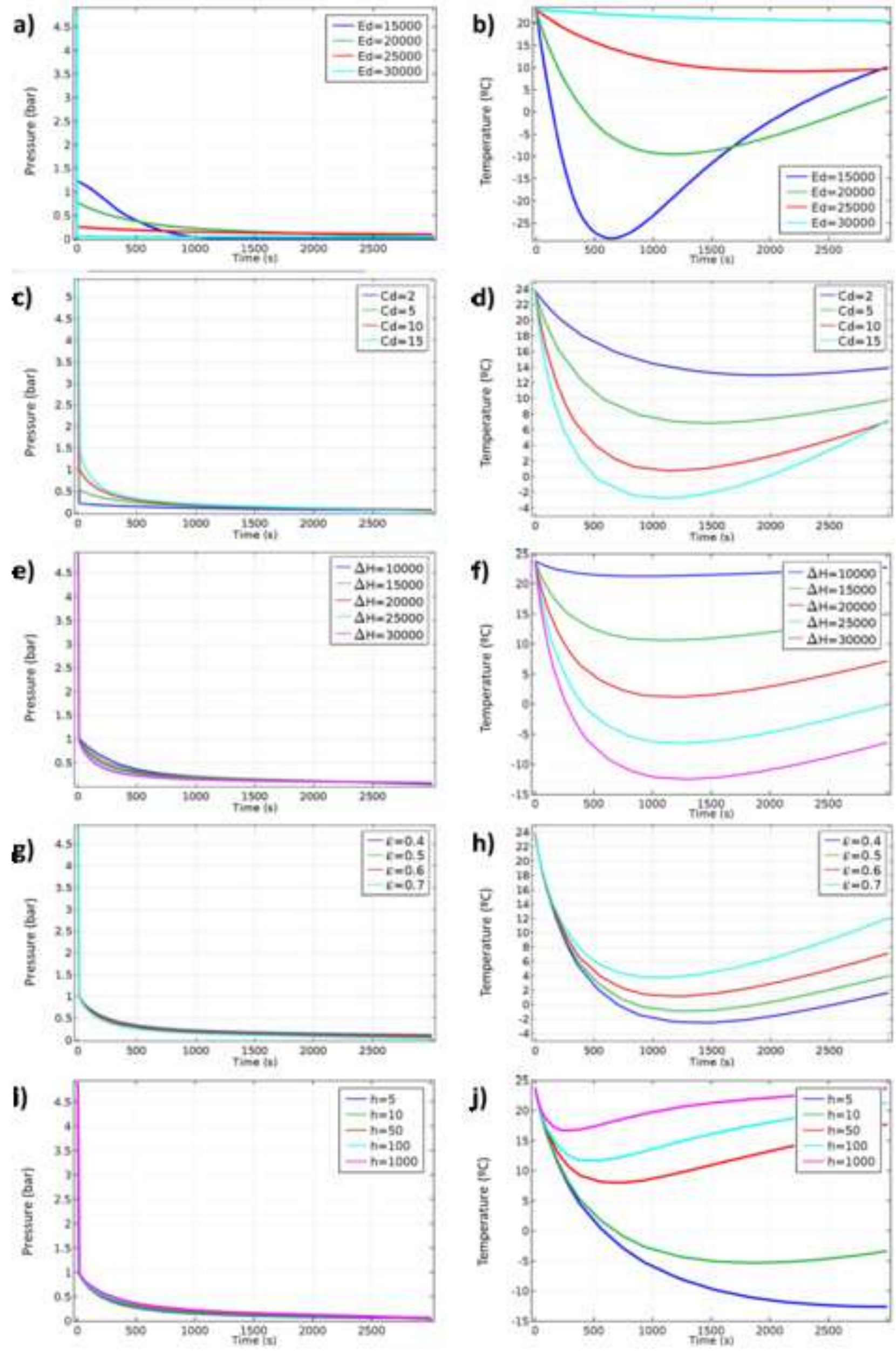

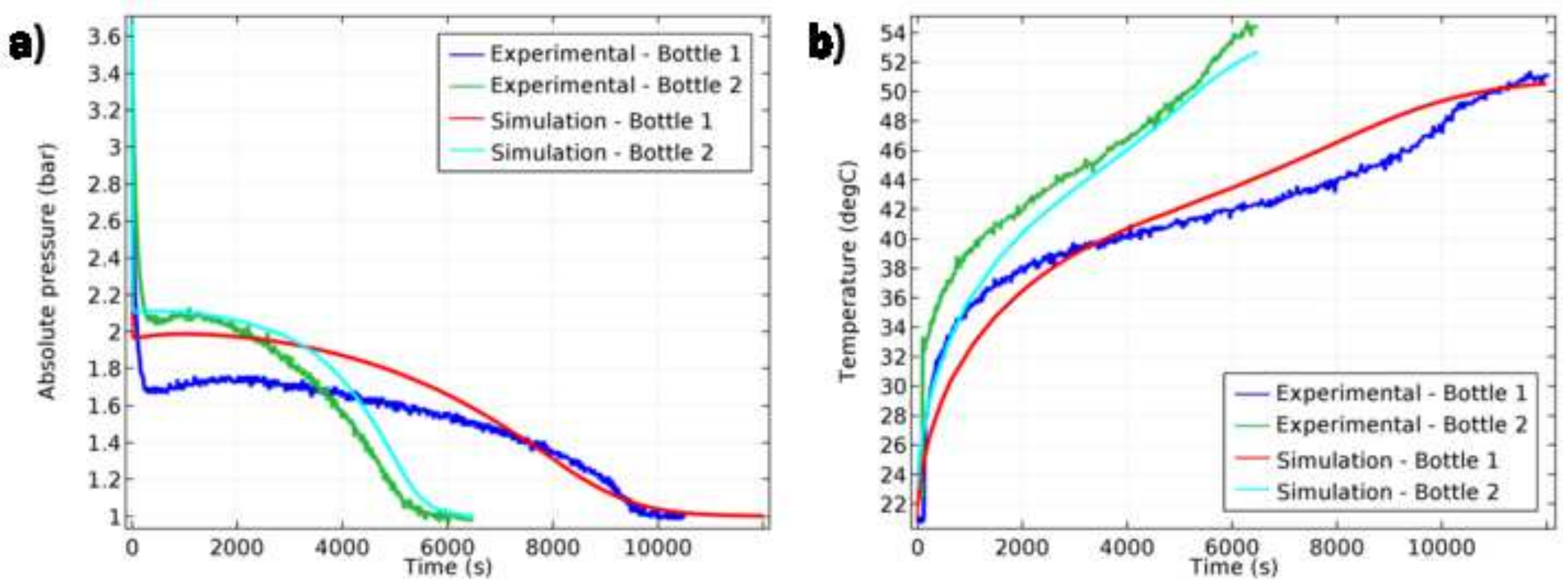